\title{
Efeitos auditivos em operadores de empilhadeira
}

\author{
Auditory effects in forklift operators
}

\author{
Adriana Lacerda ${ }^{1}$, Vanessa Gregorczyk Garofani², Leila Ribeiro² ${ }^{2}$, Jair Mendes Marques ${ }^{3}$
}

\begin{abstract}
RESUMO
Objetivo: Conhecer os efeitos, para a audição, da exposição combinada ao monóxido de carbono e ao ruído em um grupo de operadores de empilhadeira. Métodos: Realizou-se audiometria convencional em grupo controle exposto apenas ao ruído (Grupo 1) e um grupo de funcionários expostos ao monóxido de carbono e ao ruído (Grupo 2). Resultados: Observou-se um caso sugestivo de perda auditiva induzida por ruído para o grupo exposto somente ao ruído (Group 1), e cinco casos para o grupo exposto ao monóxido de carbono e ruído (Group 2). A ocorrência de mudança significativa de limiar também foi maior no Grupo 2. Observaram-se diferenças nos limiares auditivos da orelha esquerda nas frequências de 1 kHz e 8 kHz no Grupo 2, comparativamente ao Grupo 1. Conclusão: Os resultados demonstraram efeitos auditivos significativos causados pela exposição combinada ao monóxido de carbono e ao ruído em operadores de empilhadeira, em comparação ao grupo exposto somente ao ruído. Conclui-se que é imprescindível a realização dos monitoramentos ambiental, biológico e auditivo nos operadores de empilhadeira, independentemente do nível de ruído ao qual se encontram expostos.
\end{abstract}

Descritores: Efeitos do ruído; Perda auditiva/etiologia; Compostos químicos/efeitos adversos; Exposição ocupacional; Saúde do trabalhador

\section{INTRODUÇÃO}

Tem sido atribuído ao ruído, quase com absoluta exclusividade, o foco das abordagens relacionadas à saúde auditiva dos trabalhadores; sendo ele o responsável por uma das mais frequentes enfermidades profissionais irreversíveis que ocorrem em todo o mundo, a perda auditiva induzida por ruído $(\mathrm{PAIR})^{(1,2)}$. Perda Auditiva Ocupacional (PAO) é um termo frequentemente utilizado como sinônimo de PAIR; entretanto, ao se considerar PAO, é importante que se reconheça a potencialidade de outros agentes e sua possível interação com o ruído, sobre a saúde auditiva dos trabalhadores ${ }^{(2)}$.

Estudos realizados evidenciam que a presença de agentes otoagressores pode provocar perda auditiva (PA), mesmo com a presença do ruído abaixo dos limites de tolerância ${ }^{(2-6)}$. Esses estudos relatam, ainda, que a interação entre eles poderia levar

Trabalho realizado no Curso de Especialização em Audiologia Clínica da Universidade Tuiuti do Paraná - UTP - Curitiba (PR), Brasil.

(1) Doutora, Professora do Curso de Especialização em Audiologia e dos Programas de Mestrado e Doutorado em Distúrbios da Comunicação da Universidade Tuiuti do Paraná - UTP - Curitiba (PR), Brasil.

(2) Especialista em Audiologia pela Universidade Tuiuti do Paraná - UTP Curitiba (PR), Brasil; Fonoaudióloga clinica - Curitiba (PR), Brasil.

(3) Doutor, Professor dos Programas de Mestrado e Doutorado em Distúrbios da Comunicação da Universidade Tuiuti do Paraná - UTP - Curitiba (PR), Brasil.

Endereço para correspondência: Adriana Lacerda. R. Sidney Antonio Rangel Santos, 238, Santo Inácio, Curitiba (PR), Brasil, CEP: 82010-330.

E-mail: Adriana.Lacerda@utp.br

Recebido em: 17/2/2010; Aceito em: 18/5/2010 a uma PA muito maior do que aquela resultante da exposição isolada ao ruído ou ao produto químico ${ }^{(2-6)}$, possibilitando que ela progrida mesmo após o término da exposição a determinados agentes químicos ${ }^{(7)}$.

O número de contaminantes industriais investigados quanto às propriedades ototóxicas e que podem levar à PA é reduzido. Segundo o National Institute for Occupational Safety and Health $(\mathrm{NIOSH})^{(8)}$, três grupos são considerados de alta prioridade para pesquisa: solventes, asfixiantes e metais, e, atualmente, os agrotóxicos organofosforados.

$\mathrm{Na}$ família dos asfixiantes, o mais comum, encontrado no ambiente de trabalho, é o monóxido de carbono (CO). Os estudos relacionados às concentrações de $\mathrm{CO}$ ambientais ocupacionais demonstraram que as exposições a esse agente químico em lugares fechados seriam responsáveis por um grande número de acidentes ${ }^{(8)}$. O NIOSH ${ }^{(8)}$ indicou que um número representativo de trabalhadores executando diferentes funções foi intoxicado pelos vapores em combustão emitidos pelos equipamentos com motores a gasolina ou diesel.

Sabe-se que o gás emitido pelos escapamentos de veículos é frequentemente a fonte mais comum de concentrações elevadas de CO. Entretanto, diferentes tipos de equipamentos com motores a gasolina ou diesel são também responsáveis por um certo número de exposições; entre eles podemos citar as empilhadeiras ${ }^{(9)}$.

O risco de intoxicação aumenta quando o equipamento está defeituoso, sem a manutenção adequada ou quando o equipamento é utilizado em um ambiente fechado ou mal ventilado ${ }^{(10)}$.

Em humanos, a PA é um dos efeitos associados à intoxica- 
ção aguda causada pelo CO. Os efeitos da exposição crônica ao ruído e ao $\mathrm{CO}$ em humanos exercendo atividades ocupacionais são ainda pouco conhecidos ${ }^{(6)}$.

Existem evidências demonstrando que exposição ao $\mathrm{CO}$ pode potencializar a PAIR. Ele, isoladamente, não seria ototóxico, mas ocorreria um efeito de potencialização (diz-se que há potencialização quando um agente, mesmo não tóxico por si só, potencializa a toxicidade de outro agente) à ação do ruído ${ }^{(1,6,11)}$.

Por essa razão, o objetivo desta pesquisa é conhecer os efeitos na audição da exposição combinada ao o monóxido de carbono e ao ruído em um grupo de operadores de empilhadeira.

\section{MÉTODOS}

O estudo, do tipo seccional, foi aprovado pelo Comitê de Ética da Universidade Tuiuti do Paraná (UTP) sob o protocolo de número 094/2006 e autorizado pelos indivíduos por meio da assinatura do Termo de Consentimento Livre e Esclarecido.

A coleta de dados foi realizada em uma fábrica, especializada em embalagens cartonadas para produtos lácteos, culinários e bebidas, localizada na cidade de Ponta Grossa (PR), com aproximadamente 266 funcionários. Porém, obteve-se acesso a apenas dois setores da empresa, o setor de produção, com 21 funcionários, e o setor de armazenagem, com 22 funcionários.

Foram excluídos do estudo todos os trabalhadores que possuíam apenas um exame de audiometria ou que apresentavam somente o exame de referência, sem outro sequencial.

Dessa maneira, a amostra foi constituída por 34 participantes do gênero masculino, apresentando idades entre 24 e 50 anos. Os participantes foram distribuídos em dois grupos de acordo com a exposição aos agentes otoagressores:

- Grupo 1 - Ruído (N=13): funcionários do setor da impressão das embalagens (controle de qualidade) expostos somente ao ruído $\left(84,9 \mathrm{dBA} / \mathrm{L}_{\text {Aeq-8h }}\right)$, cuja função é retirar os defeitos da embalagem antes da laminação e corte.

- Grupo 2 - CO e Ruído (N=21): funcionários do setor de armazenagem (operadores de empilhadeiras) expostos ao ruído $\left(81,9 \mathrm{dBA} / \mathrm{L}_{\text {Aeq-8h }}\right)$ e ao $\mathrm{CO}$ apresentando concentrações de carboxihemoglobina $(\mathrm{COHb})$ no sangue, variando de $3 \%$ a $8 \%$. No Brasil, consideram-se como resultados normais as concentrações de $\mathrm{COHb}$ com níveis de: $3,5 \%$ para os não-fumantes e variando de $4 \%$ a $9 \%$ para os fumantes. Já para as concentrações ambientais, os limites de tolerância previstos na NR-15 são de 39 partes por milhão (ppm) ${ }^{(12)}$.

As informações sobre os resultados das avaliações ambientais relacionados ao ruído (em dBA) e ao $\mathrm{CO}$ (em ppm) foram extraídas do relatório do programa de prevenção de risco ambiental da empresa (PPRA). Assim como os resultados da avaliação biológica do $\mathrm{CO}$ (concentrações de $\mathrm{COHb}$ no sangue) foram extraídas do relatório do programa de controle médico de saúde ocupacional (PCMSO). As avaliações ambientais (ruído e $\mathrm{CO}$ ) e biológica ( $\mathrm{COHb}$ ) seguiram as recomendações das normas brasileiras e foram realizadas por higienistas industriais e enfermeiros do trabalho, respectivamente.

Para a avaliação do ruído ambiental, foi utilizado o equipamento Quest e para a dosimetria o equipamento Instrutherm, modelo DOS 500. Para a avaliação do CO, as coletas do ar ambiental foram encaminhadas para um laboratório especializado com o objetivo de realizar a cromatografia para se conhecer as concentrações de CO.

Todos os participantes apresentam uma jornada de trabalho diária de oito horas e utilizam protetores auditivos em tempo integral. Os protetores auditivos utilizados pelos participantes do Grupo 1 - Ruído são de espuma moldável e possuem um nível de redução real (NRR) de 21 dB. Já os utilizados pelo Grupo 2 - CO e Ruído são do tipo concha e possuem um NRR de $31 \mathrm{~dB}$.

A audiometria convencional periódica foi realizada utilizando os seguintes procedimentos:

- anamnese contendo informações sobre questões relacionadas ao histórico de saúde e audição, hábitos de vida e histórico ocupacional dos participantes.

- meatoscopia com o objetivo de identificar alterações no meato acústico externo que possam influenciar os achados audiológicos.

- audiometria tonal limiar, realizada segundo os critérios da portaria 19 do Ministério do Trabalho ${ }^{(12)}$. Foi utilizado o audiômetro da marca Interacoustics AD 229, fones TDH - 39, calibrados segundo os padrões internacionais ${ }^{(13)}$.

Para a análise dos resultados das audiometrias, foi utilizado critério de classificação da portaria 19, NR 7, anexo I - quadro II, Diretrizes e Parâmetros Mínimos para Avaliação e Acompanhamento da Audição em Trabalhadores Expostos a Níveis de Pressão Sonora Elevada ${ }^{(14)}$.

De acordo com essa portaria ${ }^{(14)}$, são considerados dentro dos limites aceitáveis os limiares menores ou iguais a 25 $\mathrm{dB}$ (NA); são considerados sugestivos de PAIR os audiogramas, que, nas frequências de 3 e/ou 4 e/ou 6 kHz, apresentam limiares auditivos acima de $25 \mathrm{~dB}$ (NA) e mais elevados do que nas outras frequências testadas, estando essas comprometidas ou não, tanto no teste da via aérea quanto da via óssea, em um ou em ambos os lados; são consideradas outras causas os resultados que não se enquadram na descrição anterior.

Os prontuários audiológicos dos participantes foram utilizados a fim de analisar a mudança significativa do limiar auditivo (MSL). Segundo os critérios da portaria 19 do Ministério do Trabalho ${ }^{(14)}$, considera-se MSL quando, na análise do exame de referência e do sequencial, a diferença entre as médias aritméticas dos limiares auditivos no grupo de frequências de 3, 4 e $6 \mathrm{kHz}$ iguala ou ultrapassa $10 \mathrm{~dB}(\mathrm{NA})$. Ou ainda, quando se observa uma piora igual ou maior a $15 \mathrm{~dB}(\mathrm{NA})$, em pelo menos uma das frequências de 3,4 ou $6 \mathrm{kHz}$, mesmo com limiares auditivos normais.

Para a análise estatística, foi realizada uma análise descritiva dos dados encontrados na anamnese inicial e nos achados audiológicos. As características dos grupos e a ocorrência de PAIR foram o foco da análise. Foram utilizados os testes ANOVA, teste t de Student e teste de Mann-Whitney, considerandose o nível de significância de 5\% $(0,05)$.

\section{RESULTADOS}

Na Tabela 1 são apresentados os valores referentes à estatística descritiva das variáveis: idade e tempo de serviço em função dos Grupos 1 - Ruído e 2 - CO e Ruído. 
Tabela 1. Variáveis idades e tempo de exposição ao ruído para os grupos

\begin{tabular}{lccccc}
\hline Variável & \multicolumn{2}{c}{ Grupo 1-Ruído } & \multicolumn{2}{c}{ Grupo 2 - CO e ruído } & \multirow{2}{*}{ Valor de $p$} \\
\cline { 2 - 5 } & Média & DP & Média & 7,4 & 0,06707 \\
Idade & 31,6 & 5,6 & 36,1 & 4,2 & 0,83727 \\
Tempo de ruído & 9,9 & 7,6 & 10,4 & 4 \\
\hline
\end{tabular}

Legenda: DP = desvio-padrão

Os valores referentes ao nível de ruído variaram em cinco medições de 78,9 a 87,3 dBA/ $\mathrm{L}_{\text {Aeq-8h }}\left(\right.$ Média $\left.=84,9 \mathrm{dBA} / \mathrm{L}_{\text {Aeq-8h }}\right)$ para grupo exposto ao ruído (Grupo 1) e de 78,9 a 84,5 dBA/ $\mathrm{L}_{\text {Aeq-8h }}\left(\right.$ Média $\left.=81,9 \mathrm{dBA} / \mathrm{L}_{\text {Aeq-8h }}\right)$ para grupo exposto ao $\mathrm{CO}$ e ruído (Grupo 2).

A Tabela 2 apresenta as concentrações ambientais de CO no setor de trabalho do Grupo 2 - CO e Ruído.

Tabela 2. Concentrações ambientais de monóxido de carbono (CO) no setor de trabalho do Grupo 2 - CO e Ruído

\begin{tabular}{llc}
\hline Área & \multicolumn{1}{c}{ Atividade/função } & $\begin{array}{c}\text { Concentração de CO } \\
(\mathrm{ppm})\end{array}$ \\
\hline Armazém & $\begin{array}{l}\text { Operador de empilhadeira } \\
\text { TWA } 360 \text { minutos }\end{array}$ & 56 \\
\hline Armazém & $\begin{array}{l}\text { Operador de Empilhadeira } \\
\text { TWA } 362 \text { minutos }\end{array}$ & 57 \\
\hline Armazém & $\begin{array}{l}\text { Operador de Empilhadeira } \\
\text { TWA } 360 \text { minutos }\end{array}$ & 75 \\
\hline Armazém & $\begin{array}{l}\text { Ponto fixo } \\
\text { TWA } 360 \text { minutos }\end{array}$ & 119 \\
\hline Armazém & $\begin{array}{l}\text { Ponto fixo } \\
\text { TWA } 360 \text { minutos }\end{array}$ \\
\hline Armazém & $\begin{array}{l}\text { Ponto fixo } \\
\text { TWA } 360 \text { minutos }\end{array}$ \\
\hline Armazém & $\begin{array}{l}\text { Ponto fixo } \\
\text { TWA } 360 \text { minutos }\end{array}$ \\
\hline Armazém & $\begin{array}{l}\text { Ponto fixo } \\
\text { TWA } 360 \text { minutos }\end{array}$ \\
\hline Armazém & $\begin{array}{l}\text { Ponto fixo } \\
\text { TWA } 361 \text { minutos }\end{array}$ \\
\hline Armazém & $\begin{array}{l}\text { Operador de empilhadeira } \\
\text { TWA } 360 \text { minutos }\end{array}$ \\
\hline Armazém & $\begin{array}{l}\text { Ponto fixo } \\
\text { TWA } 364 \text { minutos }\end{array}$ \\
\hline
\end{tabular}

Legenda: TWA = média ponderada pelo tempo; ppm = partes por milhão

A Tabela 3 apresenta as concentrações de carboxihemoglobina pré e pós-jornada de trabalho do Grupo 2 - CO e Ruído.

$\mathrm{O}$ teste $\mathrm{t}$ de Student foi aplicado para a comparação de concentração de carboxihemoglobina pré-jornada (média: 5\%) e pós-jornada (média: 6\%).

As Figuras 1 e 2 demonstram os limiares médios dos dois grupos de risco na orelha direita e esquerda.

A comparação dos limiares médios entre os grupos de risco foi realizada pelo teste de Mann-Whitney. Diferenças significativas foram observadas nas frequências de $1 \mathrm{kHz}$ $(\mathrm{p}=0,046125)$ e $8 \mathrm{kHz}$ na orelha esquerda $(\mathrm{p}=0,005829)$. O Grupo 2 - CO e Ruído apresentou limiares auditivos piores comparativamente ao Grupo 1 - Ruído.

Na Tabela 4 são apresentados os resultados de acordo
Tabela 3. Concentrações de carboxihemoglobina pré e pós-jornada de trabalho do Grupo 2 - CO e Ruído

\begin{tabular}{lcccc}
\hline Setor & $\begin{array}{c}\text { Pré-jornada } \\
(\%)\end{array}$ & $\begin{array}{c}\text { Pós-jornada Não-fumante } \\
(\%)\end{array}$ & Fumante \\
\hline Armazém & 0,40 & 0,54 & $\mathrm{X}$ & \\
Armazém & 0,65 & 0,80 & $\mathrm{X}$ & \\
Armazém & 0,50 & 0,65 & & $\mathrm{X}$ \\
Armazém & 0,60 & 0,50 & $\mathrm{X}$ & \\
Armazém & 0,78 & 0,90 & & $\mathrm{X}$ \\
Armazém & 0,48 & 0,50 & & $\mathrm{X}$ \\
Armazém & 0,30 & - & $\mathrm{X}$ & \\
Armazém & - & 0,80 & & $\mathrm{X}$ \\
Armazém & 0,65 & 0,73 & $\mathrm{X}$ & \\
Armazém & 0,30 & 0,60 & & $\mathrm{X}$ \\
Armazém & 0,45 & 0,55 & $\mathrm{X}$ & \\
Armazém & 0,54 & 0,65 & $\mathrm{X}$ & \\
Armazém & 0,80 & - & $\mathrm{X}$ & \\
Armazém & 0,55 & 0,90 & $\mathrm{X}$ & \\
Armazém & 0,50 & 0,53 & & $\mathrm{X}$ \\
Armazém & 0,70 & 0,75 & $\mathrm{X}$ & \\
Armazém & 0,35 & 0,90 & $\mathrm{X}$ & \\
Armazém & 0,45 & 0,50 & $\mathrm{X}$ & \\
\hline & & & &
\end{tabular}

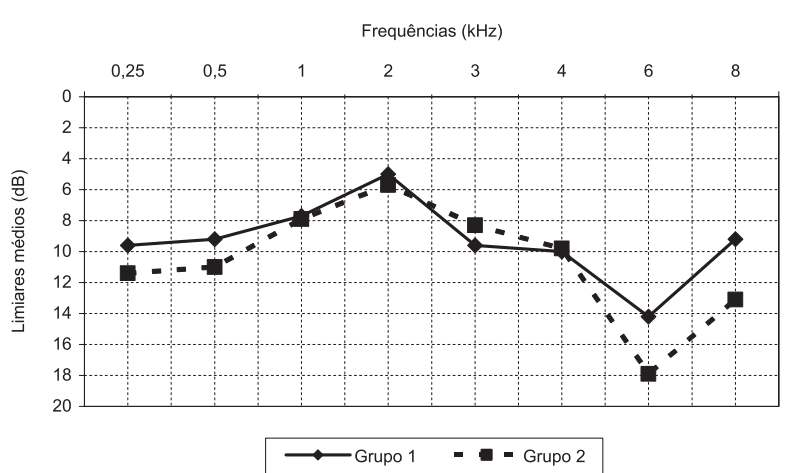

Figura 1. Limiares auditivos médios da orelha direita em função dos grupos de risco 1 (ruído) e 2 (CO e ruído)

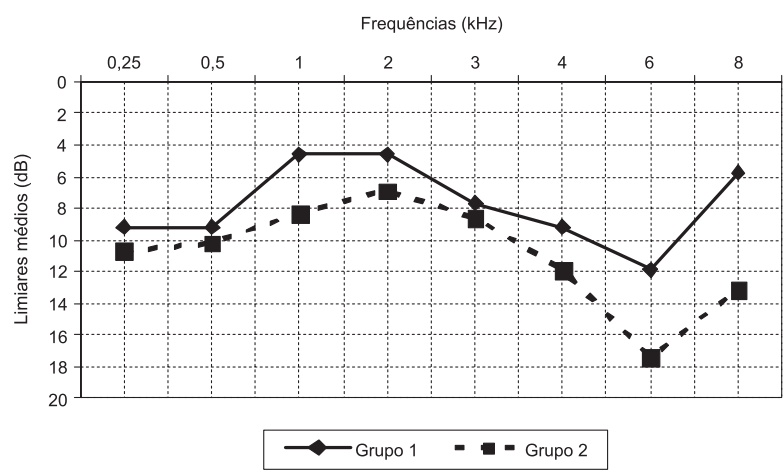

Figura 2. Limiares auditivos médios da orelha esquerda em função dos grupos de risco 1 (ruído) e 2 (CO e ruído) 
Tabela 4. Classificação da audiometria em função da frequência de ocorrências

\begin{tabular}{lcccc}
\hline Classificação & \multicolumn{4}{c}{ Número de ocorrências } \\
\cline { 2 - 5 } & \multicolumn{2}{c}{ Ruído } & \multicolumn{2}{c}{ CO e ruído } \\
\cline { 2 - 5 } & Frequência absoluta & Frequência relativa (\%) & Frequência absoluta & Frequência relativa (\%) \\
\hline Normal & 12 & 35,3 & 14 & 41,2 \\
PAIR & 1 & 2,9 & 5 & 14,7 \\
Outras causas & 0 & 0,0 & 2 & 5,9 \\
\hline
\end{tabular}

com a classificação da audiometria, segundo a Portaria 19 do Ministério do Trabalho ${ }^{(14)}$.

Com relação à MSL, observou-se que no Grupo 1 - Ruído apenas um indivíduo apresentou essa mudança unilateralmente, enquanto no Grupo 2 - CO e Ruído foram registradas 5 ocorrências, 2 unilaterais e 3 bilaterais, como demonstra a Tabela 5 .

Tabela 5. Presença de mudança significativa do limiar auditivo (MSL) em função dos grupos de risco

\begin{tabular}{lcc}
\hline Grupos de risco & \multicolumn{2}{c}{ MSL } \\
\cline { 2 - 3 } & Não & Sim \\
\hline Ruído & 12 & 1 \\
CO e Ruído & 16 & 5 \\
\hline
\end{tabular}

\section{DISCUSSÃO}

O objetivo desta pesquisa foi conhecer os efeitos, para a audição, da exposição combinada ao $\mathrm{CO}$ e ao ruído em um grupo de operadores de empilhadeira.

É importante ressaltar que os participantes do Grupo 2 CO e Ruído trabalham em um ambiente menos ruidoso $(81,9$ $\mathrm{dBA} / \mathrm{L}_{\text {Aeq-8h }}$ ) que os indivíduos do Grupo 1 - Ruído (84,9 dBA/ $\left.\mathrm{L}_{\text {Aeq-8h }}\right)$. Entretanto os participantes dos grupos analisados não apresentaram diferenças relacionadas à idade e ao tempo de exposição (Tabela 1), fatores que poderiam estar contribuindo para o agravamento auditivo ${ }^{(15,16)}$. A idade proporciona um rebaixamento nos limiares auditivos, conhecido como presbiacusia, que, associada ao ruído, causa um efeito aditivo na cóclea. Já o tempo de exposição ao ruído pode influenciar a instalação da PAIR. Nos primeiros dez a 15 anos de exposição estável ao ruído, a PAIR atinge o seu nível máximo de perda auditiva (40 dBNA para as frequências baixas e 75 dBNA para as altas) ${ }^{(17)}$.

Os resultados das avaliações ambientais da concentração de CO (Tabela 2) demonstraram que, para o setor do armazém, as concentrações ultrapassam os limites de tolerância de 39 ppm recomendados pela NR-15 ${ }^{(12)}$ do ministério do trabalho. Diferenças foram observadas $(\mathrm{p}=0,00048)$ entre as concentrações de carboxihemoglobina $(\mathrm{COHb})$ pré e pós-jornada de trabalho (Tabela 3), demonstrando que os participantes do Grupo 2 - CO e Ruído são expostos a concentrações importantes de $\mathrm{CO}$ no ambiente de trabalho, sobretudo para os participantes não fumantes.

É sabido que indivíduos normais e não fumantes, com ritmo respiratório de 20 litros por minuto e apresentando exposição ambiental de $46 \mathrm{mg} / \mathrm{m}^{3}$ de $\mathrm{CO}$ por oito horas, produzem um nível de carboxihemoglobina de $5 \%^{(18)}$. No Brasil, são considerados como resultados normais as concentrações de carboxihemoglobina com níveis de 3,5\% para os não-fumantes e variando de $4 \%$ a $9 \%$ para os fumantes ${ }^{(12)}$.

Entretanto, diferentes fatores seriam responsáveis pelas taxas de carboxihemoglobina nos indivíduos, como por exemplo, a produção endógena do $\mathrm{CO}$, a fumaça do cigarro, a exposição ao diclorometano, a carga de trabalho ou ainda a exposição a concentrações elevadas de $\mathrm{CO}$ ambiente ${ }^{(18)}$. Neste estudo, para os não fumantes, suspeita-se que as concetrações de CO ambiente poderiam estar contribuindo na elevação da carboxihemoglobina pós-jornada de trabalho.

Dessa maneira, os resultados relacionados aos achados biológicos e ambientais do CO (Tabelas 2 e 3) e aos achados audiológicos (Figuras 1 e 2) da população estudada sugerem que o $\mathrm{CO}$ poderia estar contribuindo para potencializar o efeito auditivo do ruído.

A legislação Brasileira, bem como algumas legislações internacionais, não exigem monitoramento da audição dos trabalhadores expostos a certos agentes ambientais, exceto que estejam expostos a níveis de ruído acima dos limites de exposição permitidos $(85 \mathrm{dBA})^{(12,14)}$. Porém, instituições de pesquisa como o NIOSH e a American Conference of Governmental Industrial Hygienists (ACGIH) recomendam o monitoramento da audição dos trabalhadores expostos a contaminantes químicos industriais desde $1998^{(19)}$.

Consequentemente, apesar de existir uma numerosa população de trabalhadores exposta a outros agentes otoagressores na presença de ruído de fundo, apenas uma parcela, cuja exposição ao ruído for considerada excessiva, estará incluída nos Programas de Prevenção da Perda Auditiva (PPPA).

Em geral, o PPRA apresenta o mapa de risco dos agentes isoladamente. Contudo, o monitoramento auditivo deverá levar em conta a interação entre os agentes de risco visando a prevenção, o desencadeamento e ou o agravamento da PAIR ${ }^{(20,21)}$. Um estudo $^{(2)}$ sugere que a ototoxidade dos produtos químicos não é frequentemente associada a PAO quando os trabalhadores são expostos a ruído com intensidades elevadas (acima de 85 dBA), talvez por essa razão o ruído foi há muito tempo a única causa de PA no trabalho. $\mathrm{O}$ mesmo estudo ${ }^{(2)}$ acrescenta que a audiometria sozinha não permite distinguir o efeito combinado dos contaminantes químicos e o ruído e sugere avaliações auditivas complementares, como a audiometria de altas frequências e as emissões oto acústicas.

Neste estudo, mesmo os participantes estando expostos a níveis de ruído abaixo dos limites de tolerância propostos pela NR-15 ${ }^{(12)}$ e utilizando protetores auditivos adequados para a função, registrou-se um caso de PAIR para o grupo exposto somente ao ruído $\left(84,9 \mathrm{dBA} / \mathrm{L}_{\text {Aeq-8h }}\right)$ e cinco casos para o grupo exposto ao CO e ruído $\left(81,9 \mathrm{dBA} / \mathrm{L}_{\text {Aeq-8h }}\right)$ (Tabela 4). Outro fato 
curioso é que os limiares auditivos dos participantes do Grupo 2 - CO e Ruído, mesmo dentro na normalidade, apresentam configuração em entalhe audiométrico em 3,4 ou $6 \mathrm{kHz}$, uni ou bilateral. Foram observadas diferenças nos limiares auditivos na orelha esquerda nas frequências de 1 e $8 \mathrm{kHz}$ entre os dois grupos de risco (Figura 2).

Os achados desta pesquisa vão ao encontro dos resultados demonstrados em outro estudo ${ }^{(2)}$, onde outras frequências poderiam ser afetadas após exposição prolongada aos produtos químicos, além da PA nas altas frequências ( 3 , 4 e $6 \mathrm{kHz})$. O estudo sugere que limiares anormais podem ser observados nas frequências de 2 e $8 \mathrm{kHz}$. Outros estudos ${ }^{(22,23)}$ também demonstram os efeitos da exposição combinada $\mathrm{CO}$ e ruído nas frequências de 2 e $8 \mathrm{kHz}$ e que a exposição ao ruído, mesmo abaixo dos limites de tolerância, associada ao $\mathrm{CO}$, poderia agravar ou potencializar a PAIR ${ }^{(6)}$.

Em um estudo ${ }^{(19)}$ com trabalhadores expostos ao $\mathrm{CO}$ e ruído (superior a $85 \mathrm{dBA}$ ), os resultados demonstraram que, para esse grupo, as amplitudes das emissões otoacústicas em $8 \mathrm{kHz}$ estariam reduzidas, comparativamente aos grupos expostos somente ao ruído e ao grupo sem exposição ocupacional aos dois contaminantes.

O presente estudo apresentou algumas limitações. A primeira delas foi o critério de inclusão; sem dúvida, a maior dificuldade foi encontrar participantes expostos a níveis de ruído equivalentes em uma mesma empresa. A segunda foi o desconhecimento sobre o tempo de exposição total ao ruído. E a terceira limitação estava relacionada à ausência de avaliação da vibração produzida pelas empilhadeiras; sabe-se que a vibração é um agente físico otoagressor e poderia estar contribuindo para a PA dos participantes ${ }^{(4)}$. Desse modo, os resultados deste estudo devem ser analisados com cautela; sugere-se novas pesquisas com um maior número de participantes e avaliações ambientais e biológicas de todos os agentes ambientais considerados otoagressores.

Acredita-se que um dos maiores desafios da saúde do trabalhador são os efeitos auditivos causados pelas exposições combinadas por diferentes agentes otoagressores. Assim, recomenda-se que nenhum agente otoagressor seja negligenciado e que o monitoramento audiológico de todos os trabalhadores expostos aos agentes de risco, independentemente do nível de exposição ao ruído, seja realizado.

Entretanto, a dose limite de exposição ao CO, combinada com o nível de ruído, para evitar os efeitos auditivos, é ainda desconhecida. Portanto, torna-se imprescindível que o monitoramento audiométrico dos trabalhadores seja realizado, independentemente do nível de ruído ao qual estão expostos, bem como o monitoramento ambiental e biológico da exposição ao $\mathrm{CO}$ e a outros agentes otoagressores.

\section{CONCLUSÃO}

Neste estudo, foram observados efeitos auditivos significativos no grupo com exposição combinada ao $\mathrm{CO}$ (ambiental: variando de 41 a 119 ppm/biológico: COHb média: 6\%/pósexposição) e ao ruído $\left(81,9 \mathrm{dBA} / \mathrm{L}_{\text {Aeq-8h }}\right)$ comparativamente ao grupo exposto somente ao ruído $\left(84,9 \mathrm{dBA} / \mathrm{L}_{\text {Aeq-8h }}\right)$ para a orelha esquerda. O número de casos classificados como PAIR e a ocorrência de MSL também foi maior no Grupo 2 - CO e Ruído.

\begin{abstract}
Purpose: To get to know the effects of the combined exposure to carbon monoxide and noise on hearing, in a group of forklift operators. Methods: A pure tone audiometry was carried out in a control group exposed only to noise (Group 1), and a group of employees exposed to both carbon monoxide and noise (Group 2). Results: A single case of noise-induced hearing loss was observed in the group exposed to noise (Group 1), and five cases were observed in the group exposed to both carbon monoxide and noise (Group 2). The occurrence of significant threshold change was also higher in Group 2. There was a difference between the auditory thresholds in 1 and $8 \mathrm{kHz}$ of the left ear in Group 2, when compared to Group 1. Conclusion: Results showed significant auditory effects caused by the combined exposure of forklift operators to carbon monoxide and noise, when compared to the group exposed only to noise. Thus, environmental, biological, and auditory monitoring of forklift operators is indispensable, regardless the level of noise to which they are exposed.
\end{abstract}

Keywords: Noise effects; Hearing loss/etiology; Chemical compounds/adverse effects; Occupational exposure; Health occupational

\title{
REFERÊNCIAS
}

1. Mello AP, Waismann W. Exposição ocupacional ao ruído e químicos industriais e seus efeitos no sistema auditivo: revisão de literatura. Arq Int Otorrinolaringol. 2004;8(3):226-34.

2. Morata TC. Chemical exposure as a risk factor for hearing loss. J Occup Environ Med. 2003;45(7):676-82.

3. Jacob LCB, Alvarenga KF, Morata TC. Os efeitos da exposição ocupacional ao chumbo sobre o sistema auditivo: uma revisão de literatura. Rev Bras Otorrinolaringol. 2002;68(4):564-9.
4. Fernandes M. Estudo dos efeitos auditivos e extra-auditivos da exposição ocupacional a ruído e vibração. Rev Bras Otorrinolaringol. 2002;68(5):705-13.

5. Lacerda A, Leroux T, Gagn JP. The combined effect of noise and carbon monoxide on hearing thresholds of exposed workers. J Acoust Soc Am. 2005;117(4):2481.

6. Lacerda A, Leroux T, Morata T. Efeitos ototóxicos da exposição ao monóxido de carbono: uma revisão. Pró-Fono. 2005;17(3):403-12. 
7. Fuente A, McPherson B. Organic solvents and hearing loss: The challenge for audiology. Int J Audiol. 2006;45(7):367-81.

8. Center for Disease Control and Prevention (CDC). National Institute for Occupational Safety and Health (NIOSH). Carbon Monoxide Poisoning. April, 2009. [acesso em 08 de novembro de 2010]. Disponível em: http://www.cdc.gov/co/default.htm

9. Fawcett TA, Moon RE, Fracica PJ, Mebane GY, Theil DR, Piantadosi CA. Warehouse workers' headache. Carbon monoxide poisoning from propane-fueled forklifts. J Occup Med. 1992;34(1):12-5.

10. Roberge B. Evaluation and control of carbon monoxide exposure from propane-fuelled forklifts. Appl Occup Environ Hyg. 1998;13(3):183-91.

11. Fechter LD, Chen GD, Rao D. Chemical asphyxiants and noise. Noise Health. 2002;4(14):49-61

12. Brasil. Ministério do Trabalho e Emprego. NR-15 - Atividades e operações insalubres (115.000.6). CLT capítulo V, título II. Diário Oficial da União; 8 de junho de 1978.

13. American National Standards Institute 1999. Maximum permissible ambient noise levels for audiometric test rooms. ANSI/ASA S3.1-1999 (R2008). New York; 1999.

14. Brasil. Ministério do Trabalho. Secretaria de Segurança e Saúde no Trabalho. Portaria N 19 de 9 de abril de 1998. Estabelece diretrizes e parâmetros mínimos para a avaliação e o acompanhamento da audição dos trabalhadores expostos a níveis de pressão sonora elevados. NR-7 Programa de Controle Médico de Saúde Ocupacional. Diário Oficial da União; 30 dezembro de 1994. p. 21278.
15. Rabinowitz PM, Galusha D, Ernst CD, Slade MD. Audiometric "early flags" for occupational hearing loss. J Occup Environ Med. 2007;49(12):1310-6.

16. Prince MM, Stayner LT, Smith RJ, Gilbert SJ. A re-examination of risk estimates from the NIOSH Occupational Noise and Hearing Survey (ONHS). J Acoust Soc Am. 1997;101(2):950-63. Comment in: J Acoust Soc Am. 1998;103(5 Pt 1):2734-9.

17. Gonçalves CGO. Saúde do trabalhador: da estruturação à avaliação de programas de preservação auditiva. São Paulo: Rocca; 2009.

18. Scarino A, Tardif R. Modélisation de l'exposition au monoxyde de carbone. Rapport du l'Institut de recherche Robert-Sauvé en santé et sécurité du travail - IRSST. 2004. p.27.

19. Lacerda AB. Effets de l'exposition chronique au monoxyde de carbone et au bruit sur l'audition [tese]. Montreal (CA): Université de Montreal; 2009.

20. Morata TC, Franks JR, Dunn DE. Unmet needs in occupational hearing conservation. Lancet. 1994;344(8920):479.

21. Morata TC, Little MB. Suggested guidelines for studying the combined effects of occupational exposure to noise and chemicals on hearing. Noise Health. 2002;4(14):73-87.

22. Shahbaz Hassan M, Ray J, Wilson F. Carbon monoxide poisoning and sensorineural hearing loss. J Laryngol Otol. 2003;117(2):134-7.

23. Morata TC, Dunn DE, Kretschmer LK, Lemasters GK, Keith RW. Effects of occupational exposure to organic solvents and noise on hearing. Scand J Work Environ Health. 1993;19(4):245-54. 